\title{
Distinctive Blood Eosinophilic Phenotypes and Cytokine Patterns in Eosinophilic Esophagitis, Inflammatory Bowel Disease and Airway Allergy
}

\author{
Marianne Johnsson ${ }^{a}$ Mogens Bove ${ }^{d}$ Henrik Bergquist ${ }^{b}$ Mikael Olsson ${ }^{e}$ \\ Sven Fornwall ${ }^{f}$ Karin Hassel ${ }^{f}$ Agnes E. Wold ${ }^{a}$ Christine Wennerås ${ }^{a, c}$ \\ Departments of a Clinical Bacteriology, ${ }^{\mathrm{b}}$ ENT, Head and Neck Surgery, and ${ }^{\mathrm{C}}$ Hematology and Coagulation, \\ Sahlgrenska University Hospital, Göteborg, Departments of ${ }^{\mathrm{d}}$ ENT, Head and Neck Surgery, and ${ }^{\mathrm{e}}$ Internal Medicine, \\ NÄL Hospital, Trollhättan, and ${ }^{\mathrm{f}}$ Norrmalm Primary Health Care Center, Skövde, Sweden
}

\section{Key Words}

Cytokines $\cdot$ Eosinophils $\cdot$ Inflammatory bowel disease $\cdot$

Eosinophilic esophagitis $\cdot$ Flow cytometry

\begin{abstract}
Blood eosinophil numbers may be elevated in allergy, inflammatory bowel disease and eosinophilic esophagitis. The aim of this study was to examine whether circulating eosinophils display distinct phenotypes in these disorders and if different patterns of eosinophilic chemoattractants exist. Blood eosinophils from patients with symptomatic eosinophilic esophagitis (EoE; $n=12$ ), ulcerative colitis $(n=8)$, airway allergy $(n=10)$ and healthy controls $(n=10)$ were enumerated and their surface markers analyzed by flow cytometry. Plasma levels of pro-eosinophilic cytokines were quantified in parallel. Data were processed by multivariate pattern recognition methods to reveal disease-specific patterns of eosinophil phenotypes and cytokines. EoE patients had higher numbers of eosinophils with enhanced expression of CD23, CD54, CRTH2 and CD11C and diminished CCR3 and CD44 expression. Plasma CCL5 was also increased in EoE. Although allergic patients had increased interleukin (IL)-2, IL-3, IL-5 and granulocyte macrophage colony-stimulating factor plasma
\end{abstract}

concentrations, their blood eosinophil phenotypes were indistinguishable from those of healthy controls. Decreased eosinophilic expression of CD11b, CD18, CD44 and CCR3, but no distinctive pattern of eosinophil chemoattractants, characterized ulcerative colitis. We propose that eosinophils acquire varying functional properties as a consequence of distinct patterns of activation signals released from the inflamed tissues in different diseases.

Copyright $\odot 2011$ S. Karger AG, Basel

\section{Introduction}

Eosinophilic granulocytes accumulate in the tissues in several inflammatory disorders, including allergies, inflammatory bowel disease $[1,2]$ and the recently described condition eosinophilic esophagitis (EoE) [3]. In EoE, eosinophils invade the mucosa of the esophagus, a part of the gastrointestinal tract that is normally devoid of these cells. Symptoms include difficulty in swallowing food, which may even result in food impaction, heartburn, abdominal pain, nausea and vomiting. Both children and adults are afflicted by the disease whose cause is unknown [4]. Many patients are sensitized to food and

\section{KARGER \\ Fax +4161306 1234 \\ E-Mail karger@karger.ch}

www.karger.com (c) 2011 S. Karger AG, Basel

1662-811X/11/0036-0594\$38.00/0

Accessible online at:

www.karger.com/jin
Dr. Christine Wennerås

Department of Clinical Bacteriology

Sahlgrenska University Hospital

Box 7193, SE-402 34 Göteborg (Sweden)

Tel.+4631342 4784, E-Mail christine.wenneras@microbio.gu.se 
Table 1. Patient characteristics

\begin{tabular}{|c|c|c|c|c|c|c|}
\hline \multirow{2}{*}{$\begin{array}{l}\text { Diagnostic } \\
\text { category }\end{array}$} & \multirow{2}{*}{$\begin{array}{l}\text { Patients } \\
\mathrm{n}\end{array}$} & \multirow{2}{*}{$\begin{array}{l}\text { Median age } \\
\text { years }\end{array}$} & \multirow{2}{*}{$\begin{array}{l}\text { Females } \\
\%\end{array}$} & \multirow{2}{*}{$\begin{array}{l}\text { Disease-specific } \\
\text { medication }\end{array}$} & \multicolumn{2}{|c|}{ Topical inhalant allergy medication } \\
\hline & & & & & steroid & $\beta_{2}$-stimulant \\
\hline $\mathrm{EoE}$ & 12 & $41(17-62)$ & 25 & $\begin{array}{l}\text { proton pump inhibitor } 8 \% \\
\text { carbamazepine } 8 \% \\
\text { no treatment } 83 \%\end{array}$ & $17 \%$ & $0 \%$ \\
\hline UC & 8 & $42(19-76)$ & 38 & $\begin{array}{l}\text { mesalazine (sulfa) } 25 \% \\
\text { no treatment } 62 \%\end{array}$ & $12 \%$ & $12 \%$ \\
\hline AA & 10 & $30(24-50)$ & 100 & $\begin{array}{l}\text { sodium cromoglycate } 10 \% \\
\text { oral antihistamine } 20 \% \\
\text { no treatment } 60 \%\end{array}$ & $0 \%$ & $30 \%$ \\
\hline $\mathrm{HC}$ & 10 & $39(22-61)$ & 90 & not applicable & $0 \%$ & $0 \%$ \\
\hline
\end{tabular}

Figures in parentheses are ranges.

inhalant allergens and children improve when started on hypoallergenic elemental diets; whether this holds true for adults is less clear [5].

Eosinophils mature and differentiate in the bone marrow under the influence of granulocyte macropahge colony-stimulating factor (GM-CSF), interleukin (IL)-3 and IL-5 [6]. In the healthy state, newly produced eosinophils pass via the bloodstream to their final destination, primarily the gut mucosa, but also to the thymus, spleen and lymph nodes [7]. Eosinophils constitute $<5 \%$ of the white blood cells in the circulation in healthy individuals, but this proportion may increase in parasite infections, as well as in allergic and other inflammatory conditions characterized by eosinophilic involvement.

Eosinophils are recruited from the bloodstream into the tissues by locally produced chemokines and other chemotactic factors. This traffic occurs under steadystate conditions and is further enhanced during inflammation. Factors that may attract eosinophils include the cytokines/chemokines eotaxin-1, -2 and -3 , IL-5, CCL5 (RANTES), the tripeptide formyl-methionyl-leucyl-phenylalanine released by metabolically active bacteria and damaged mitochondria, and platelet-activating factor [8]. In the tissue, eosinophils may recognize and be activated by airborne allergens [9-11], bacteria [12] and necrotic epithelial cells [13]. Their role in the pathogenesis of asthma, inflammatory bowel disease and EoE is unclear, and both pathogenic [14, 15] and protective functions [16] have been suggested. In addition, eosinophils participate in tissue remodeling [17] and may be engaged in immune regulation [18].
As eosinophils appear to be able to fulfill several functions, it is possible that their phenotype in the blood may vary in different inflammatory disorders. This may, in turn, depend on the mixture of cytokines and other inflammatory mediators released from the afflicted tissues that regulate the production and release of eosinophils from the bone marrow, as well as the activation and trafficking of eosinophils in the bloodstream. Here, we have investigated the phenotype of blood eosinophils in patients with ongoing inflammatory diseases characterized by tissue eosinophil infiltration, namely allergic airway disease, ulcerative colitis (UC) and EoE. We also examined the blood levels of eosinophil chemoattractants and growth factors in the same individuals. The aim was to define disease-specific patterns of eosinophil activation, which could shed some light on the pathogenic processes characterizing these diseases and perhaps be used for diagnostic purposes.

\section{Materials and Methods}

\section{Patients}

We investigated 40 adult individuals: 12 with symptomatic EoE $(n=12), 8$ with symptomatic UC, 10 with symptomatic airway allergy (AA; asthma and/or allergic rhinitis) and 10 healthy controls (HC) (table 1).

Patients with EoE were recruited at the Head and Neck Surgery Department of either NÄL Hospital or Sahlgrenska University Hospital, Sweden. Ten out of $12 \mathrm{EoE}$ patients had typical endoscopic features of EoE including trachealization, furrows, white specks, mucosal shredding and/or strictures and all had experienced dysphagia and/or food impaction. Biopsies were

J Innate Immun 2011;3:594-604 
Table 2. Monoclonal antibodies used for flow cytometric analyses

\begin{tabular}{|c|c|c|c|c|}
\hline $\mathrm{CD}$ & Molecule & Clone & Isotype & Fluorochrome \\
\hline CD4 & co-receptor for MHC II & RPA-T4 & $\operatorname{IgG}_{1, \kappa}$ & APC \\
\hline CD9 & binds integrins, triggers platelets & M-L13 & $\operatorname{IgG}_{1, \kappa}$ & $\mathrm{PE}$ \\
\hline CD11b & forms integrin Mac-1 (CR3) w CD18 & D12 & $\operatorname{IgG}_{2 \mathrm{a}, \kappa}$ & $\mathrm{PE}$ \\
\hline CD11c & forms integrin p150,95 (CR4) w CD18 & B-ly6 & $\operatorname{IgG}_{1, \kappa}$ & APC \\
\hline CD16 & Fc $\gamma$ RIIIb, IgG-R & $3 \mathrm{G} 8$ & $\operatorname{IgG}_{1 \mathrm{a}, \kappa}$ & FITC \\
\hline CD23 & FceRII, IgE-R & EBVCS-5 & $\operatorname{IgG}_{1, \kappa}$ & APC \\
\hline CD25 & IL-2R $\alpha$-chain & $2 \mathrm{~A} 3$ & $\operatorname{IgG}_{1, \kappa}$ & APC \\
\hline CD40 & binds CD154 (CD40L) on T cells & $5 \mathrm{C} 3$ & $\operatorname{IgG}_{1, \kappa}$ & APC \\
\hline CD44 & binds hyaluronic acid & G44-26 & $\operatorname{IgG}_{2 \mathrm{~b}, \kappa}$ & APC \\
\hline CD49d & VLA-4 $\alpha$-chain & 9F10 & $\operatorname{IgG}_{1, \kappa}$ & $\mathrm{PE}$ \\
\hline CD184 & CXCR4 & $12 \mathrm{G} 5$ & $\operatorname{IgG}_{2 \mathrm{a}, \kappa}$ & APC \\
\hline CD193 & CCR3 & $5 \mathrm{E} 8$ & $\operatorname{IgG}_{2 \mathrm{~b}, \kappa}$ & $\mathrm{PE}$ \\
\hline \multirow[t]{3}{*}{ CD294 } & CRTH2 (PGD 2 receptor $2, \mathrm{DP} 2)$ & BM16 & rat $\operatorname{IgG}_{2 a}$ & Alexa Fluor 647 \\
\hline & formyl peptide receptor 2 (FPR2, FPRL1) & 304405 & $\operatorname{IgG}_{2 b}$ & APC \\
\hline & major basic protein (MBP P1) & AHE-2 & $\operatorname{IgG}_{1}$ & unconjugated \\
\hline
\end{tabular}

gathered from the distal, middle and proximal part of the esophagus and from the fundus ventriculi and the duodenum in all EoE patients. All EoE patients had a peak count of $>20$ eosinophils per microscopic high-power field in at least one biopsy [3]: the median peak number of eosinophils was $40 /$ high-power field (range 20-80). Eosinophilic infiltration of the mucosa due to concurrent gastroesophageal reflux disease was ruled out by lack of response to proton pump inhibitor therapy and/or normal acid levels according to 24-hour $\mathrm{pH}$ monitoring. More than half (7/12) had a history of inhalant allergy; none were smokers.

UC patients $(n=8)$ were recruited at the Gastroenterology Unit at NÄL Hospital, Trollhättan, Sweden. Five of the patients were new cases, and 3 were previously diagnosed and displayed endoscopic signs of flare-up. The grade of inflammation was scored according to the Baron system (median score 2.0, range 1.5-3.0). The regional distribution in the intestine was as follows: segmental colitis $(n=1)$, proctitis $(n=2)$, proctosigmoiditis $(n=$ $2)$, extensive colitis $(n=1)$ and total colitis $(n=2)$.

Patients with symptomatic AA were recruited at Norrmalm Primary Health Care Center, Skövde, Sweden, and had IgE-dependent allergy, as evidenced by Phadiatop (Phadia AB, Uppsala, Sweden) or skin prick tests. Patients taking systemic corticosteroids were excluded, and topical corticosteroids were discontinued prior to sampling (table 1). HC were recruited among friends and colleagues. No physical examination was done. The subjects declared themselves to be healthy and were not allowed to have any symptoms or inflammatory processes at the time of sampling. None were endoscoped. The study was approved by the Research Ethics Committee of the Medical Faculty at the University of Go- thenburg, Gothenburg, Sweden, and written informed consent was obtained from all participants.

\section{Study Setup}

All study participants donated $12 \mathrm{ml}$ of EDTA-anticoagulated venous blood on one occasion. The maximal time between drawing of blood and flow cytometry analyses was $24 \mathrm{~h}$; blood samples were kept at room temperature, and control experiments revealed that this did not affect eosinophil phenotypes or cytokine levels in plasma (data not shown). Eosinophils were quantified using an automated cell counter (ADVIA 2120i; Siemens Medical Solutions Diagnostics, Tarrytown, N.Y., USA). After removal of erythrocytes by repeated hypotonic lysis, leukocytes were washed once in Krebs-Ringer-glucose buffer [19] and analyzed immediately by flow cytometry for eosinophil phenotypes. Plasma was stored at $-20^{\circ} \mathrm{C}$ for later assessment of eosinophil-activating cytokines.

\section{Flow Cytometry}

Unfractionated leukocytes were incubated with panels of antibodies conjugated with FITC, PE, APC, or Alexa Fluor 647 (table 2) for $15 \mathrm{~min}$ at room temperature, washed and analyzed by 4-color fluorescence using a FACSCanto II ${ }^{\mathrm{TM}}$ Flow Cytometer (BD Biosciences, San Diego, Calif., USA). FluoroSpheres (DakoCytomation, Glostrup, Denmark) were employed as calibration controls. All antibodies were from BD Biosciences except for antiKORSA-3544 (Beckham Coulter, Inc., Fullerton, Calif., USA) and anti-FPR2 antibody (R\&D Systems, Minneapolis, Mich., USA). Intracytoplasmic staining of major basic protein and CXCR4 was performed using the IntraStain kit (DakoCytomation). 
Eosinophils were identified as a population with high side scatter and low CD16 expression, as opposed to neutrophils that were CD16 ${ }^{\text {hi }}$. To confirm the absence of eosinophils in the CD16 ${ }^{\text {hi }}$ population, CD16 ${ }^{\text {hi }}$ cells were sorted (FACSAria cell sorter; BD Biosciences), cytospins prepared (Cytospin; Shandon Scientific Co. Ltd., London, UK), stained by eosin and examined in the light microscope (100 cells counted). No eosinophils were found in this preparation.

Multiple isotype controls are inadequate for multicolor staining, as true controls require that one parameter be changed at a time. Instead, the 'fluorochrome minus one' technique was used, in which positive events are discriminated from negative events not by a single isotype-based threshold or unstained cells, but by multiple controls characterized by sequential removal of each fluorochrome-labeled antibody used in the multicolor stain [20]. However, for intracellular stains, an irrelevant isotype-matched antibody (anti-CD19; BD Biosciences) was used to monitor background staining. Data were collected using FACSDiva ${ }^{\mathrm{TM}}$ Software 6.0 (BD Biosciences); 100,000 events were acquired and analyzed using the software Flow Jo 7.2.2 (Tree Star Inc., Ashland, Oreg., USA). Data were expressed as median fluorescence intensity.

\section{Cytokines}

Plasma levels of IL-2, IL-3, IL-5, GM-CSF, CCL5 (RANTES) and CCL11 (eotaxin-1) were determined using the Cytometric Bead Array (BD Biosciences) and analyzed by flow cytometry. Data were analyzed using the FCAP Array software (BD Biosciences). CCL26 was detected using Human Eotaxin-3 Duo Set ELISA (R\&D Systems Ltd., Abingdon, UK). The limits of detection were: $11 \mathrm{pg} / \mathrm{ml}$ for IL-2, $0.3 \mathrm{pg} / \mathrm{ml}$ for IL-3 and $1.1 \mathrm{pg} / \mathrm{ml}$ for IL-5, $0.8 \mathrm{pg} / \mathrm{ml}$ for eotaxin, $0.002 \mathrm{pg} / \mathrm{ml}$ for CCL5, $0.2 \mathrm{pg} / \mathrm{ml}$ for GMCSF and $30 \mathrm{pg} / \mathrm{ml}$ for CCL26.

\section{Statistics}

Multivariate analysis for pattern recognition was performed using the SIMCA-P statistical package (Umetrics, Umeå, Sweden). 'Partial least squares projections to latent structures' with discriminant analysis (PLS-DA) [21] was used to see if models could be created in which study persons clustered into their respective diagnostic categories based on eosinophilic activation markers in blood or plasma concentrations of cytokines. The fitness of the models was evaluated by cross-validation. Flow cytometry and cytokine data were log transformed prior to analysis when deemed appropriate by the software based on skewness of data. Cytokine values below the detection limit were set to 0.5 times this limit. Findings derived from the PLS-DA models were further analyzed using the nonparametric Kruskal-Wallis method (GraphPad Prism 5.0 software; GraphPad, San Diego, Calif., USA). Correlations were assayed using the Spearman rank correlation test (GraphPad Prism).

\section{Results}

To test the hypothesis that the various eosinophil-associated diseases differed with respect to eosinophil phenotypes and patterns of eosinophilic chemoattractants in peripheral blood, all data were analyzed by the multivar- iate technique of PLS-DA. This method models the relationship between a large set of study variables $(\mathrm{X})$, in this case eosinophilic activation markers or plasma cytokine levels, and outcome variables (Y), in this case, diagnostic categories. An advantage compared to traditional regression analyses is that all variables can be analyzed simultaneously since 'mass significance' is not a problem and variables are allowed to be interdependent.

We first examined eosinophil surface marker expression in the four diagnostic groups EoE, UC, AA and HC. However, no model could be obtained that was able to separate all four groups. This failure was the result of a large overlap between the $\mathrm{HC}$ and allergic groups (data not shown). Univariate analysis confirmed that eosinophils from controls and allergics did not differ significantly with respect to any of the measured parameters (data not shown). Therefore, we constructed a new model based on EoE, UC and HC, excluding AA study persons. This model showed a good fit (fig. la) and was able to separate the individuals into the three groups EoE, UC and $\mathrm{HC}$, respectively (fig. 1b). Figure $1 \mathrm{c}$ shows which variables were responsible for this separation; variables situated close to the position of a certain diagnostic category tended to be higher in this group of patients, while variables distant from the diagnostic category tended to be lower, compared to the other disease categories. According to the obtained PLS-DA model, the following features characterized eosinophils retrieved from the blood of patients with EoE, relative to the other eosinophil-dominated inflammatory conditions and to HC: high absolute numbers of blood eosinophils, relative numbers of eosinophils and enhanced eosinophilic expression of CRTH2, CD11c, CD23 (low-affinity IgE receptor) and CD54 (ICAM-1) (fig. 1c). Conversely, the CCR3 and CD44 variables were positioned close to the category of $\mathrm{HC}$, signifying that eosinophils derived from the two patient groups EoE and UC had reduced levels of these two receptors, compared with healthy persons (fig. 1c). Finally, CD11b and CD18 were positioned directly opposite to the UC category, indicating that these markers were relatively decreased in UC patients compared to EoE and HC (fig. 1c). Variables situated in the middle of the figure lacked the power to discriminate between the conditions included in the model and were not analyzed further (fig. 1c).

Variables identified by the pattern recognition method PLS-DA, important for the discrimination of EoE from UC and $\mathrm{HC}$, respectively, were further selected for analysis using the univariate non-parametric KruskalWallis test (fig. 2). These analyses confirmed that CD23, 
Fig. 1. Clustering of patients into clinical categories EoE, UC and HC based on eosinophil counts and surface markers, using 'projection onto latent structures' with 'discriminant analysis' (PLS-DA) modeling. a Fitness of model is indicated by $\mathrm{R}^{2} \mathrm{VY}_{\text {cum }}$ (black columns), the cumulative sum of squares of all $\mathrm{Y}$ values (diagnostic categories) and $\mathrm{X}$ values (surface markers and eosinophil numbers) explained by the model, and $\mathrm{Q}^{2} \mathrm{VY}_{\text {cum }}$ (hatched columns), the fraction of the total variation in the $\mathrm{Y}$ variable that can be predicted by the sum of the X variables. $\mathbf{b}$ Separation of patients according to diagnosis into HC (lower left quadrant, labeled by crosses), UC (upper part of diagram, open diamonds) and EoE (right part, black boxes). Axes are labeled by score vectors $\mathrm{t}[1]$ and $\mathrm{t}[2]$, synthetic variables constructed from the original $\mathrm{X}$ variables to contain as much information as possible of relevance for modeling the $\mathrm{Y}$ variables. c Eosinophil data (X variables) that contribute to the segregation of the clinical groups (Y variables). The position of the $\mathrm{X}$ variables corresponds to the position of the individuals in panel $b$, i.e., variables close to the EoE diagnosis marker (number of eosinophils, \% eosinophils, CD23, CRTH2, CD11c, CD54) are higher in these patients, while variables close to the HC diagnosis marker (CCR3 and CD44) are generally higher in $\mathrm{HC}$ than in the two disease categories. Weight variables $\mathrm{w}^{\cdot} \mathrm{c}[1]$ and $\mathrm{w}^{\cdot} \mathrm{c}[2]$ show which variables contribute to the PLS-DA model; the weight for X variables is denoted ' $w$ ', while the weight for $Y$ variables is denoted ' $c$ ', depicted in the same figure.
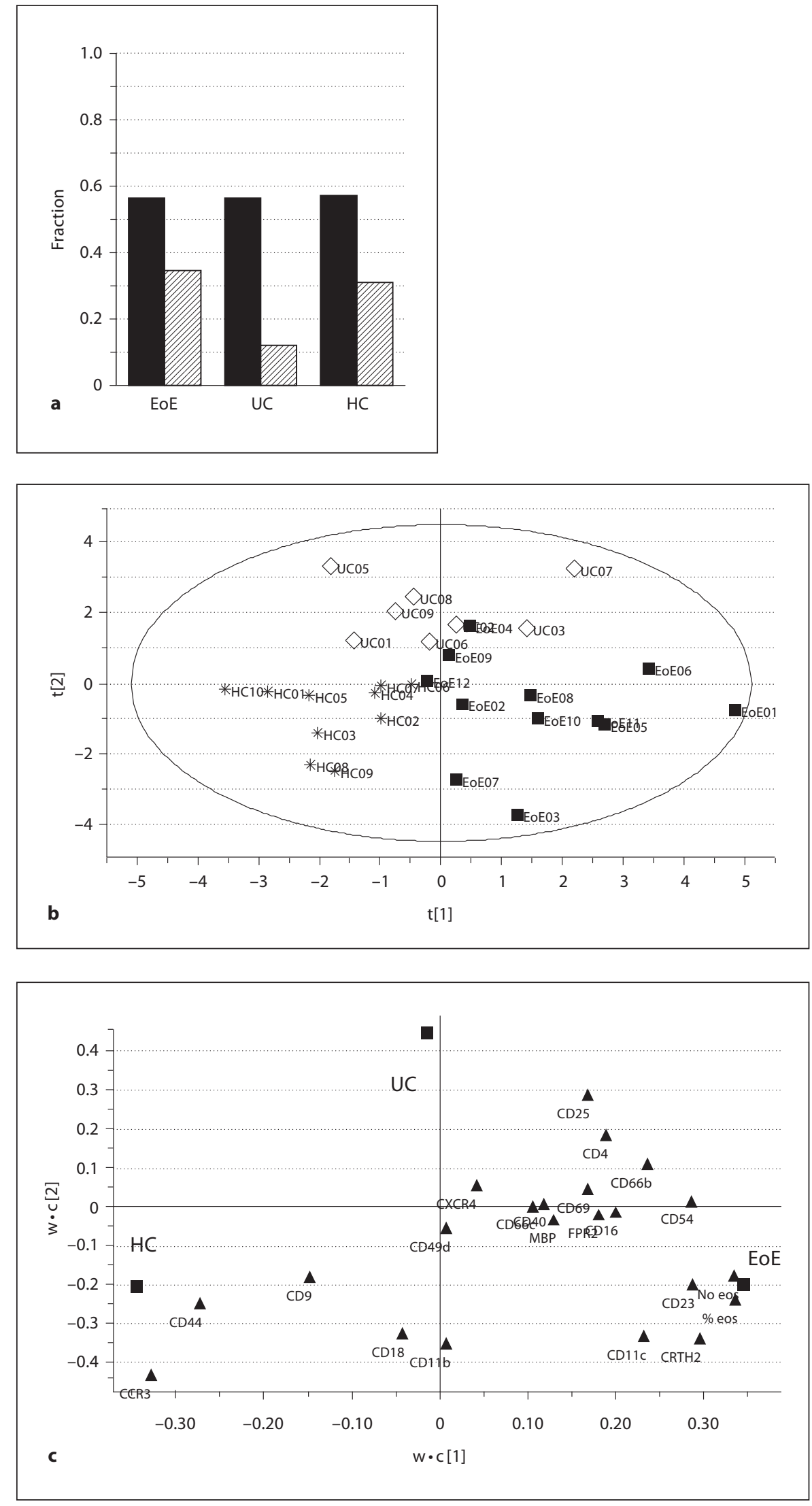


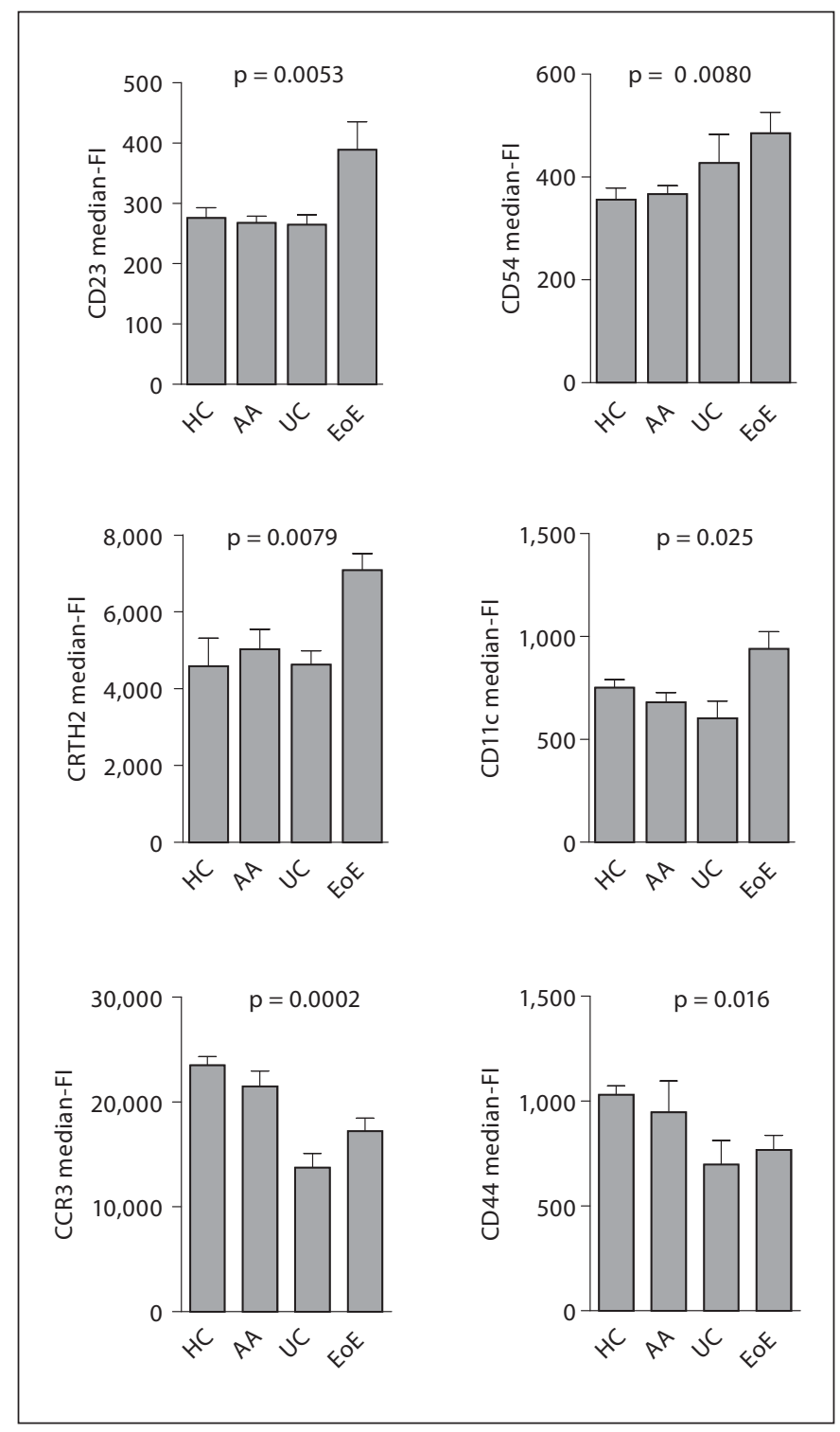

Fig. 2. Eosinophilic expression of surface markers. Expression of CD23, CD54, CRTH2, CD11c, CD44 and CCR3 was assessed by FACS and expressed as median fluorescence intensity (medianFI). Columns indicate the mean and SEM for HC, AA, UC and EoE patients. Differential expression of surface markers between the clinical groups was analyzed by the Kruskal-Wallis test; the $\mathrm{p}$ values are indicated in the figure.

CD54, CRTH2, CD11c, CCR3 and CD44 were differently expressed on eosinophils isolated from blood of patients belonging to the studied categories. Thus, eosinophils from EoE patients had increased expression of CD23, CD54, CRTH2 and CD11c, while CCR3 and CD4 were more strongly expressed on eosinophils from $\mathrm{HC}$ and $\mathrm{AA}$ patients (fig. 2). Moreover, EoE patients had significantly
Table 3. Absolute and relative eosinophil numbers in peripheral blood

\begin{tabular}{llrllr}
\hline & \multicolumn{2}{l}{ Eosinophils, $\times 10^{9} / 1$} & & \multicolumn{2}{l}{ Eosinophils, \% } \\
\cline { 3 - 3 } \cline { 6 - 7 } & median & $\begin{array}{l}\text { 25th-75th } \\
\text { percentile }\end{array}$ & & median & $\begin{array}{r}\text { 25th-75th } \\
\text { percentile }\end{array}$ \\
\hline EoE & 0.34 & $0.17-0.51$ & & 4.6 & $2.3-7.9$ \\
UC & 0.080 & $0.060-0.17$ & & 0.92 & $0.81-2.4$ \\
AA & 0.15 & $0.10-0.34$ & & 2.1 & $1.5-3.6$ \\
HC & 0.096 & $0.059-0.19$ & & 1.5 & $0.89-3.0$ \\
p value & & \multicolumn{2}{c}{0.013} & & \multicolumn{2}{c}{0.0074} \\
\hline
\end{tabular}

${ }^{1}$ Kruskal-Wallis test.

higher levels of absolute as well as relative blood eosinophil counts compared to the other groups (table 3). CD11b and CD18 expression was lower in UC patients relative to the other categories but did not reach statistical significance (data not shown).

Next, we studied the plasma levels of cytokines of relevance for the maturation and release of eosinophils from the bone marrow into the bloodstream and their recruitment into the inflamed tissues. First, we examined whether PLS-DA could generate a model capable of discriminating between the four clinical groups. This was not possible; the UC group overlapped too much with the HCs to permit the construction of a valid model. Instead, we generated a model composed of $\mathrm{HC}, \mathrm{EoE}$ and $\mathrm{AA}$ study persons. This model had sufficient explanatory capacity (fig. 3a) and was able to separate the three groups from one another reasonably well (fig. 3b). However, as seen in the observation plot, some patients with AA clustered together with the $\mathrm{HC}$, and the EoE patients were widely spread out (fig. 3b). Figure $3 c$ illustrates that CCL26 and CCL11 projected close to the HC, CCL5/ RANTES was adjacent to the EoE disease category, and GM-CSF, IL-3, IL-2 and IL-5 were positioned close to the AA category. Univariate analyses largely confirmed these findings: EoE patients displayed higher average levels of CCL5 (RANTES) than all other groups, and elevated levels of GM-CSF typified airway allergics (fig. 4). IL-2 and IL-3 were similarly increased in the blood of EoE and AA subjects (fig. 4). Finally, although IL-5 was higher in airway allergics, it was elevated in all inflammatory conditions, e.g., in EoE and UC as well (fig. 4).

We also performed a series of univariate correlation tests (Spearman rank test) to unravel interdependence between the parameters identified to have power to dis- 
Fig. 3. Clustering of patients by PLS-DA based on blood cytokine levels. The model permitted discrimination between EoE, AA and HC. a Model characteristics where $\mathrm{R}^{2} \mathrm{VY}_{\text {cum }}$ (black columns) signifies the cumulative sum of squares of all the $Y$ values (diagnostic categories) and $\mathrm{X}$ values (cytokines) explained by the model, and $\mathrm{Q}^{2} \mathrm{VY}_{\text {cum }}$ (hatched columns) is the fraction of the total variation in the $\mathrm{Y}$ variable that can be predicted by the sum of the $\mathrm{X}$ variables. b Separation of patients according to diagnosis into HC (tight cluster on the left, labeled by crosses), AA (lower part of diagram, open triangles) and EoE (upper part of diagram, mainly to the right, black boxes). Axes are labeled by score vectors $t[1]$ and $\mathrm{t}[2]$, synthetic variables constructed from the original $\mathrm{X}$ variables to contain as much information as possible of relevance for modeling the $\mathrm{Y}$ variables. c Cytokine data (X variables) that contribute to the segregation of the clinical groups (Y variables). The position of the variables corresponds to the position of the individuals in panel b, i.e., CCL5 adjacent to the EoE diagnostic category is higher in these patients, while GM-CSF, IL-2, IL-3 and IL-5 are close to the AA diagnosis, and hence, high in these patients. Weight variables $\mathrm{w} \cdot \mathrm{c}[1]$ and $\mathrm{w} \cdot \mathrm{c}[2]$ show which variables contribute to the PLS-DA model; the weight for X variables is denoted ' $w$ ', while the weight for Y variables is denoted ' $c$ ', depicted in the same figure.
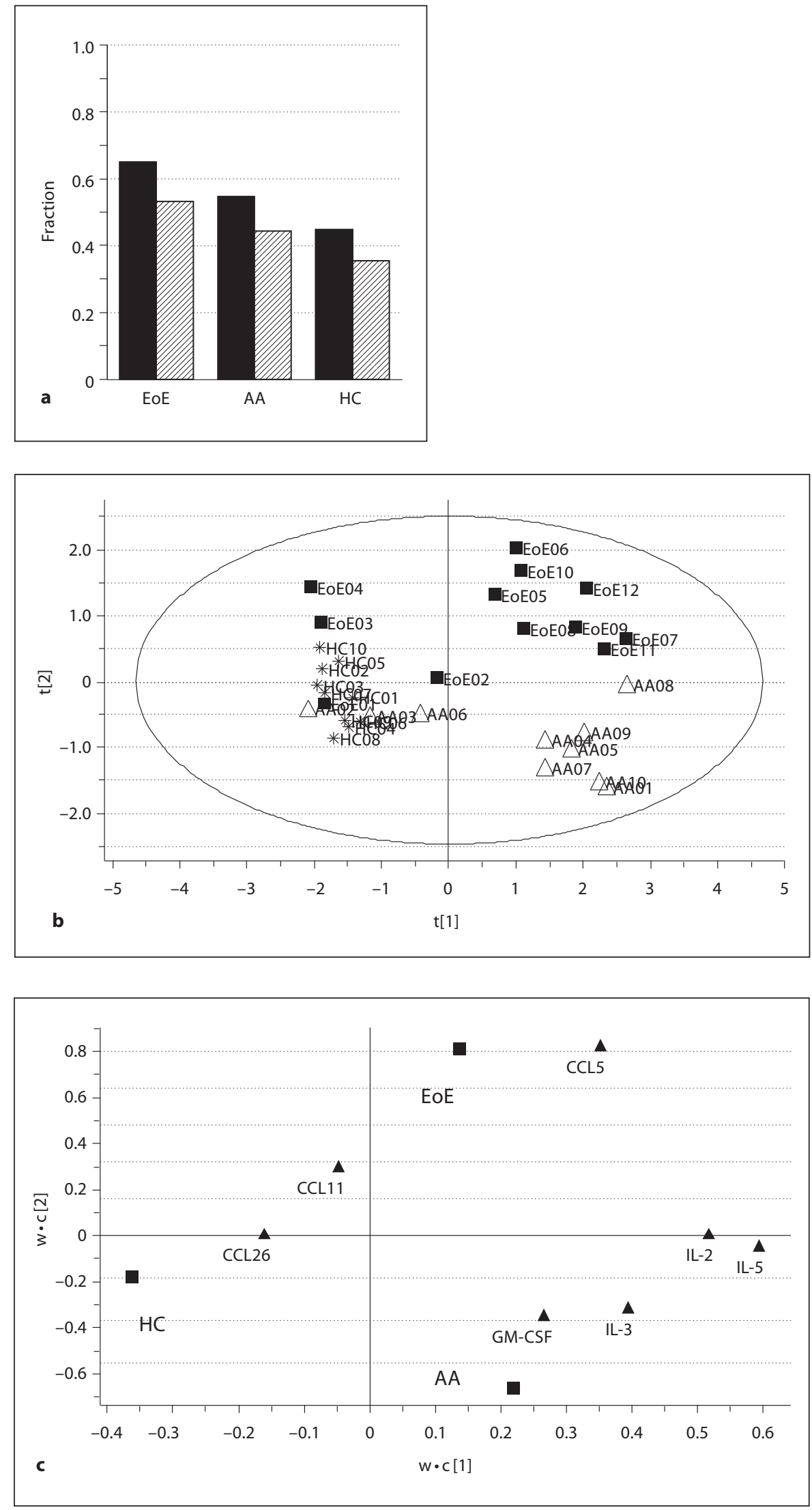



Fig. 4. Plasma levels of cytokines involved in eosinophil production, maturation and chemotaxis in $\mathrm{HC}, \mathrm{AA}, \mathrm{UC}$ and EoE patients. Difference in cytokine levels according to clinical group was tested using the Kruskal-Wallis test. Arithmetic means +1 SEM are shown.

criminate between the studied clinical categories. Each diagnostic group was analyzed separately to avoid the creation of false correlations that depended on differences between the groups. First, we examined whether the surface markers that were relatively increased in EoE, e.g., CRTH2, CD23, CD11c and CD54, or relatively decreased in EoE, CCR3 and CD44, were correlated. A strong positive correlation was found between $\mathrm{CD} 23$ (low-affinity IgE receptor) and CD54 (ICAM-1; r = 0.82, $p=0.001$ ), while none of the other markers were significantly correlated to one another. Next, we examined correlations between absolute or relative eosinophil numbers in blood and the identified eosinophil markers and cytokines. The percentage of eosinophils in the circulation of EoE patients correlated positively with the expression of CD9 on eosinophils ( $\mathrm{r}=0.59, \mathrm{p}=0.041)$ and negatively with the levels of CCL11 (eotaxin-1) in plasma $(r=-0.61$, $\mathrm{p}=0.037$ ). In fact, a highly significant negative correlation was seen between the percentage of eosinophils in the circulation and plasma CCL11 levels when EoE and $\mathrm{UC}$ patients were analyzed jointly $(\mathrm{r}=-0.60, \mathrm{p}=0.0049)$, but not when AA patients and/or $\mathrm{HC}$ were included in the analysis. No other significant correlations were uncovered between eosinophil numbers, surface markers and cytokines for the other clinical conditions.

\section{Discussion}

Here, we studied surface marker expression on circulating eosinophils as well as eosinophil maturation factors and chemoattractants in the blood of patients with three different diseases characterized by eosinophilic tissue infiltration: EoE, UC and AA. Surface marker expression was analyzed by flow cytometry using nonfractionated leukocytes in order to avoid spurious activation caused by immunomagnetic purification of eosinophils. All data were analyzed using the multivariate 'pattern recognition' method PLS-DA to reveal disease-specific eosinophilic phenotypes and/or cytokines, followed by univariate analysis of variables identified to have discriminatory power. Our findings indicate that blood eosinophil phenotypes could be used to distinguish patients with EoE and UC from one another and from HC. In contrast, plasma cytokine patterns discriminated between EoE patients, allergics and healthy persons.

Of the studied diseases, EoE was characterized by the highest absolute and relative eosinophil numbers in the blood. Eosinophils are produced in the bone marrow in response to GM-CSF, IL-3 and IL-5 [6]. An elevated plasma GM-CSF level was restricted to airway allergics, whereas IL-3 and IL-5 were raised in the plasma of EoE patients and airway allergics alike. IL-2 was increased in the blood of both allergics and EoE patients, suggestive of T-cell involvement in these conditions. Unexpectedly, correlation analyses failed to demonstrate any significant associations between the levels of bone marrow stimulatory cytokines and absolute numbers of eosinophils in the blood of any patient category. This lack of association 
may depend on the presence of additional substances that promote the production of eosinophils and their release from the bone marrow. A candidate chemoattractant is prostaglandin $\mathrm{D}_{2}$, whose receptor CRTH2 was overexpressed by eosinophils in EoE. Activation of CRTH2 by prostaglandin $\mathrm{D}_{2}$ boosts the release of eosinophils from the bone marrow and primes the cells to become more sensitive to the effects of other chemoattractants [22]. Mast cells are the main producers of prostaglandin $\mathrm{D}_{2}$ and appear in greater numbers in the esophagus of EoE patients [3]. An alternate explanation for our inability to demonstrate a positive association between eosinophil numbers and cytokines is that an eosinophilopoietin such as IL-5 primarily exerts its effects on eosinophil progenitor cells that express the IL-5 receptor, and not on mature eosinophils that have lost the receptor [6]. In fact, anti-IL-5 therapy of EoE patients has been tested and resulted in much reduced blood eosinophil levels but only halved tissue eosinophil counts, which may explain the modest clinical improvement that was achieved [23].

Among EoE patients, we found raised levels of a single chemokine in the blood relative to healthy persons and the other disease categories, namely CCL5 (RANTES). CCL5 is a strong eosinophil chemoattractant [24], which is produced in the esophagus in EoE [25]. CCL26 (eotaxin-3) has been regarded as a signature chemokine of EoE, previously found in both the esophagus and the circulation [26]; however, in our patient material, we found neither CCL26 nor CCL11 (eotaxin-1) to be increased in the blood of EoE subjects or any of the other patient groups. Actually, an inverse correlation was documented regarding the percentage of circulating eosinophils and CCL11 levels in the blood for EoE and UC patients, indicative of consumption of this chemokine. Moreover, we observed lower surface expression of CCR3, the receptor for both CCL11 and CCL26 in these same patient groups, which we interpret to reflect internalization of the receptor following activation by ligand(s) [27]. Our data also suggest that CCR3CCL11 appear to be of importance in the eosinophilic disorders affecting the gastrointestinal tract, e.g., the esophagus and colon, but not the airways, since CCR 3 expression and CCL11 levels were similar in allergics and in HC. Lastly, we uncovered a positive correlation between raised percentage of blood eosinophils and upregulated CD9 expression in EoE, which may be linked to the capacity of CD9 activation to prolong eosinophilic survival [28].

A large number of surface molecules on blood eosinophils were upregulated in EoE. CD23, the low-affinity IgE receptor, whose two isoforms a and b are expressed by eosinophils [29], was expressed in increased amounts.
This is interesting in view of recent findings indicating that local production of IgE may occur in the esophagus of patients with EoE [30]. CD23 positively regulates IgE production [31] but may also facilitate antigen presentation of allergen complexed to IgE [32], which may contribute to the perpetuation of chronic inflammation to minute amounts of allergen or other exogenous antigen implicated in the pathogenesis of EoE. ICAM-1 was also upregulated on EoE eosinophils. The ligands for ICAM-1 are the $\beta_{2}$-integrins Cd11aCD18 (LFA-1) and Cd11bCD18 (Mac-1) found on many types of leukocytes. Importantly, we discovered an unexpected and strong correlation between ICAM-1 and CD23 on EoE eosinophils. ICAM-1 expression by eosinophils has been shown to promote their interaction with T cells [33]. Close cellular encounters are required for immunomodulation, and eosinophils are able to skew T cells towards Th1 and Th2 types of responses [34]. Moreover, eosinophils are endowed with antigen-presenting ability [34]. This might indicate an immunoregulatory role for eosinophils in the chronic inflammatory condition of EoE, whose cause is presently unknown, although a triggering allergen or other exogenous agent may be at the root of it.

Blood eosinophils also bore markers of activation in UC. Eosinophils derived from the intestinal mucosa of patients with UC live longer and release eosinophil peroxidase more readily than do eosinophils from Crohn's disease patients [35]. We found some resemblance between blood eosinophils from UC and EoE patients, e.g., a shared diminished expression of CCR3 and CD44, the receptor for hyaluronic acid. In accordance, Lampinen et al. [35] have described increased surface expression of CD44 by eosinophils in the quiescent phase of UC. Maybe downregulation of CCR3 and CD44 is a pattern characteristic of eosinophils homing to inflamed gastrointestinal mucosa. Decreased binding to hyaluronic acid in the tissue might endow eosinophils with greater migratory potential. A tendency towards decreased expression of the $\beta_{2}$-integrin Mac-1 (CD11bCD18) has previously been reported in UC patients compared to HC [36]; a similar but nonsignificant tendency was noted here. The infiltration of eosinophils into the intestinal wall in UC has been considered to be a negative prognostic factor [37] as well as a favorable one [16]. The eosinophil does have destructive potential since it can release potent granule proteins that can catalyze the creation of toxic metabolites [18]. On the other hand, we have shown that eosinophils exposed to damaged intestinal cells in vitro produce fibroblast growth factor 2 [13], suggesting that they may be involved in the healing process. 
None of our AA patients had severe allergic manifestations at the time of sampling, which may explain why we could not document an allergy-specific phenotype. Our results are in agreement with those of Bochner [38], who emphasizes the difficulty of defining unique eosinophilic phenotypes in allergic diseases. This finding was quite surprising, considering that the AA patients exhibited the highest plasma levels of all but one (CCL5) of the eosinophil-related cytokines in our patient material, namely IL-2, IL-3, IL-5 and GM-CSF. All these cytokines have been shown to enable diapedesis of eosinophils across endothelial layers and prime eosinophils to become more responsive to other stimuli [38]. However, we were unable to unmask any correlations between any of these cytokines and any of the examined eosinophil activation markers. Inhibition of IL-5 abrogates the release of eosinophils into the blood and reduces their extravasation into the bronchial tree in patients with AA [39]. In fact, anti-IL-5 treatment has been shown to improve asthma control in selected patients who are resistant to corticosteroids $[14,40]$. Although the use of systemic corticosteroid was not allowed in our study, a few patients used topical steroids (inhaled or nasal); the limited number of patients did not permit us to analyze the impact of topical steroids on eosinophil phenotypes. Hence, we cannot exclude that topical steroid treatment caused the relative inertness of blood eosinophils in patients with AA.

A limitation of our study was that we did not examine eosinophil phenotypes in the inflamed tissues, but only the phenotypes of eosinophils 'en route' to the tissues. However, our hypothesis was that eosinophil phenotypes would vary, depending on the underlying disease, already in the circulation before having reached the tissues and become modulated by tissue-specific factors. We were able to confirm that blood eosinophil phenotypes differed according to the associated disease, which suggests that eosinophils receive disease-specific signals during their differentiation in the bone marrow and/or in the blood, presumably to meet specific demands of the particular condition. Furthermore, our findings might also be used as an aid in the diagnosis of inflammatory diseases in the future, e.g., to indicate which patients need more invasive diagnostic procedures such as biopsies.

In summary, eosinophils displayed diverse immunophenotypic profiles in patients with EoE, UC and AA, respectively. This suggests that eosinophils receive different signals of activation in these diseases. Hopefully, these findings can be exploited to facilitate the diagnosis of eosinophil-related diseases and increase the knowledge regarding their pathophysiology.

\section{Acknowledgements}

This work was funded by grants from the Swedish Research Council (K2010-57X-14180-09-3), LUA-ALF (71580), Cancer and Allergy Foundation, Health and Medical Care Committee of the Regional Executive Board of Region Västra Götaland (96490), Council Th. Bergh Foundation, and Inga-Britt and Arne Lundberg Research Foundation.

\section{Disclosure Statement}

The authors declare that they have no competing commercial or financial interests.

\section{References}

1 Bischoff SC, Wedemeyer J, Herrmann A, Meier PN, Trautwein C, Cetin Y, Maschek H, Stolte M, Gebel M, Manns MP: Quantitative assessment of intestinal eosinophils and mast cells in inflammatory bowel disease. Histopathology 1996;28:1-13.

-2 Carvalho AT, Elia CC, de Souza HS, Elias PR, Pontes EL, Lukashok HP, de Freitas FC, Lapa e Silva JR: Immunohistochemical study of intestinal eosinophils in inflammatory bowel disease. J Clin Gastroenterol 2003;36:120125 .

\footnotetext{
-3 Furuta GT, Liacouras CA, Collins MH, Gupta SK, Justinich C, Putnam PE, Bonis P, Hassall E, Straumann A, Rothenberg ME: Eosinophilic esophagitis in children and adults: a systematic review and consensus recommendations for diagnosis and treatment. Gastroenterology 2007;133:1342-1363.

-4 Straumann A, Simon HU: Eosinophilic esophagitis: escalating epidemiology? J Allergy Clin Immunol 2005;115:418-419.

$\checkmark 5$ Spergel JM: Eosinophilic esophagitis in adults and children: evidence for a food allergy component in many patients. Curr Opin Allergy Clin Immunol 2007;7:274-278.

66 Gauvreau GM, Ellis AK, Denburg JA: Haemopoietic processes in allergic disease: eosinophil/basophil development. Clin Exp Allergy 2009;39:1297-1306.
}

\footnotetext{
7 Kato M, Kephart GM, Talley NJ, Wagner JM, Sarr MG, Bonno M, McGovern TW, Gleich GJ: Eosinophil infiltration and degranulation in normal human tissue. Anat Rec 1998; 252:418-425.

-8 Lampinen M, Carlson M, Hakansson LD, Venge P: Cytokine-regulated accumulation of eosinophils in inflammatory disease. Allergy 2004;59:793-805.

$\checkmark 9$ Plotz SG, Traidl-Hoffmann C, Feussner I, Kasche A, Feser A, Ring J, Jakob T, Behrendt $\mathrm{H}$ : Chemotaxis and activation of human peripheral blood eosinophils induced by pollen-associated lipid mediators. J Allergy Clin Immunol 2004;113:1152-1160.
} 
10 Redvall E, Bengtsson U, Wenneras C: Responsiveness of eosinophils to aeroallergens may be independent of atopic status. Scand J Immunol 2008;67:377-384.

- 11 Svensson L, Redvall E, Bjorn C, Karlsson J, Bergin AM, Rabiet MJ, Dahlgren C, Wenneras C: House dust mite allergen activates human eosinophils via formyl peptide receptor and formyl peptide receptor-like 1. Eur J Immunol 2007;37:1966-1977.

$>12$ Svensson L, Wenneras C: Human eosinophils selectively recognize and become activated by bacteria belonging to different taxonomic groups. Microbes Infect 2005;7:720 728.

13 Stenfeldt AL, Wenneras C: Danger signals derived from stressed and necrotic epithelial cells activate human eosinophils. Immunology 2004;112:605-614.

- 14 Nair P, Pizzichini MM, Kjarsgaard M, Inman MD, Efthimiadis A, Pizzichini E, Hargreave FE, O’Byrne PM: Mepolizumab for prednisone-dependent asthma with sputum eosinophilia. N Engl J Med 2009;360:985993.

$>15$ Shichijo K, Makiyama K, Wen CY, Matsuu M, Nakayama T, Nakashima M, Ihara M, Sekine I: Antibody to eosinophil cationic protein suppresses dextran sulfate sodium-induced colitis in rats. World J Gastroenterol 2005; 11:4505-4510.

$\checkmark 16$ Heatley RV, James PD: Eosinophils in the rectal mucosa. A simple method of predicting the outcome of ulcerative proctocolitis? Gut 1979;20:787-791.

17 Flood-Page P, Menzies-Gow A, Phipps S, Ying S, Wangoo A, Ludwig MS, Barnes N, Robinson D, Kay AB: Anti-IL-5 treatment reduces deposition of ECM proteins in the bronchial subepithelial basement membrane of mild atopic asthmatics. J Clin Invest 2003 112:1029-1036.

- 18 Jacobsen EA, Taranova AG, Lee NA, Lee JJ: Eosinophils: singularly destructive effector cells or purveyors of immunoregulation? J Allergy Clin Immunol 2007;119:1313-1320.

19 Svensson L, Dahlgren C, Wenneras C: The chemoattractant Trp-Lys-Tyr-Met-Val-DMet activates eosinophils through the formyl peptide receptor and one of its homologues, formyl peptide receptor-like 1 . J Leukoc Biol 2002;72:810-818.

20 Roederer M: Compensation in flow cytometry; in Robinson JP: Current Protocols in Cytometry. New York, John Wiley and Sons, 2002, suppl 22, pp 1.14.11-11.14.20.
21 Eriksson L, Antti H, Gottfries J, Holmes E, Johansson E, Lindgren F, Long I, Lundstedt T, Trygg J, Wold S: Using chemometrics for navigating in the large data sets of genomics, proteomics, and metabonomics (gpm). Anal Bioanal Chem 2004;380:419-429.

22 Heinemann A, Schuligoi R, Sabroe I, Hartnell A, Peskar BA: Delta 12-prostaglandin $\mathrm{J}_{2}$, a plasma metabolite of prostaglandin $\mathrm{D}_{2}$, causes eosinophil mobilization from the bone marrow and primes eosinophils for chemotaxis. J Immunol 2003;170:47524758 .

23 Straumann A, Conus S, Grzonka P, Kita H, Kephart G, Bussmann C, Beglinger C, Smith DA, Patel J, Byrne M, Simon HU: Anti-interleukin-5 antibody treatment (mepolizumab) in active eosinophilic oesophagitis: a randomised, placebo-controlled, double-blind trial. Gut 2010;59:21-30.

24 Alam R, Stafford S, Forsythe P, Harrison R, Faubion D, Lett-Brown MA, Grant JA: RANTES is a chemotactic and activating factor for human eosinophils. J Immunol 1993;150: 3442-3448

25 Straumann A, Bauer M, Fischer B, Blaser K, Simon HU: Idiopathic eosinophilic esophagitis is associated with a $\mathrm{T}(\mathrm{H}) 2$-type allergic inflammatory response. J Allergy Clin Immunol 2001;108:954-961.

26 Blanchard C, Wang N, Stringer KF, Mishra A, Fulkerson PC, Abonia JP, Jameson SC, Kirby C, Konikoff MR, Collins MH, Cohen MB, Akers R, Hogan SP, Assa'ad AH, Putnam $\mathrm{PE}$, Aronow BJ, Rothenberg ME: Eotaxin-3 and a uniquely conserved gene-expression profile in eosinophilic esophagitis. J Clin Invest 2006;116:536-547.

27 Zimmermann N, Rothenberg ME: Receptor internalization is required for eotaxin-induced responses in human eosinophils. J Allergy Clin Immunol 2003;111:97-105.

28 Kim JT, Gleich GJ, Kita H: Roles of CD9 molecules in survival and activation of human eosinophils. J Immunol 1997;159:926-933.

29 Abdelilah SG, Bouchaïb L, Morita M, Delphine A, Marika S, André C, Monique C: Molecular characterization of the low-affinity IgE receptor Fc epsilonRII/CD23 expressed by human eosinophils. Int Immunol 1998;10:395-404.

-30 Vicario M, Blanchard C, Stringer KF, Collins $\mathrm{MH}$, Mingler MK, Ahrens A, Putnam PE, Abonia JP, Santos J, Rothenberg ME: Local B cells and IgE production in the oesophageal mucosa in eosinophilic oesophagitis. Gut 2010;59:12-20.
31 Bonnefoy JY, Gauchat JF, Life P, Graber P, Aubry JP, Lecoanet-Henchoz S: Regulation of IgE synthesis by CD23/CD21 interaction. Int Arch Allergy Immunol 1995;107:40-42.

-32 van der Heijden FL, Joost van Neerven RJ, van Katwijk M, Bos JD, Kapsenberg ML: Serum-IgE-facilitated allergen presentation in atopic disease. J Immunol 1993;150:36433650 .

33 Hansel TT, De Vries IJ, Carballido JM, Braun RK, Carballido-Perrig N, Rihs S, Blaser K, Walker C: Induction and function of eosinophil intercellular adhesion molecule-1 and HLA-DR. J Immunol 1992;149:2130-2136.

34 Akuthota P, Wang HB, Spencer LA, Weller PF: Immunoregulatory roles of eosinophils: a new look at a familiar cell. Clin Exp Allergy 2008;38:1254-1263

35 Lampinen M, Backman M, Winqvist O, Rorsman F, Ronnblom A, Sangfelt P, Carlson M: Different regulation of eosinophil activity in Crohn's disease compared with ulcerative colitis. J Leukoc Biol 2008;84:13921399.

>36 Coppi LC, Thomazzi SM, de Ayrizono ML, Coy CS, Fagundes WJ, Goes JR, Franchi GC, Jr., Nowill AE, Montes CG, Antunes E, Ferraz JG: Comparative study of eosinophil chemotaxis, adhesion, and degranulation in vitro in ulcerative colitis and Crohn's disease. Inflamm Bowel Dis 2007;13:211-218.

37 Ahrens R, Waddell A, Seidu L, Blanchard C, Carey R, Forbes E, Lampinen M, Wilson T, Cohen E, Stringer K, Ballard E, Munitz A, Xu H, Lee N, Lee JJ, Rothenberg ME, Denson L, Hogan SP: Intestinal macrophage/epithelial cell-derived CCL11/eotaxin-1 mediates eosinophil recruitment and function in pediatric ulcerative colitis. J Immunol 2008;181: 7390-7399.

38 Bochner BS: Systemic activation of basophils and eosinophils: markers and consequences. J Allergy Clin Immunol 2000;106:S292S302.

39 Flood-Page PT, Menzies-Gow AN, Kay AB, Robinson DS: Eosinophil's role remains uncertain as anti-interleukin-5 only partially depletes numbers in asthmatic airway. Am J Respir Crit Care Med 2003;167:199-204.

40 Haldar P, Brightling CE, Hargadon B, Gupta S, Monteiro W, Sousa A, Marshall RP, Bradding P, Green RH, Wardlaw AJ, Pavord ID: Mepolizumab and exacerbations of refractory eosinophilic asthma. N Engl J Med 2009;360:973-984. 